\title{
Deriving Multiple Representation Database: A Model Generalisation Approach
}

\author{
J. Boodala ${ }^{\text {a, } *, \text { O. Dikshit }}{ }^{\text {a }}$ N. Balasubramanian ${ }^{\text {a }}$ \\ ${ }^{a}$ Geoinformatics, Department of Civil Engineering, Indian Institute of Technology Kanpur, Kanpur - 208016, Uttar Pradesh, India. \\ jaggubreddy@gmail.com,onkar@iitk.ac.in,nagaraj@iitk.ac.in \\ * Corresponding author
}

\begin{abstract}
The main aim of the research being conducted is to automatically derive multiple representation database through model generalisation. In this regard, this paper presents a detailed methodology of the derivation process of multiple representation database. The topographic information of three different levels of detail (LoDs) viz., LoD1 $(1: 10,000)$, LoD2 $(1: 25,000)$ and LoD3 $(1: 250,000)$ are considered for this research. The abstract LoDs, i.e., LoD2 and LoD3 are derived automatically by model generalisation. The method for linking corresponding features in all these LoDs is shown and thus deriving multiple representation database. The major component of the proposed methodology is the model generalisation process. The design of the model generalisation process which includes generalisation operators, classification hierarchies, geometric constraints, functional hierarchies and topological constraints are discussed in this article. The proposed methodology is currently in the implementation stage.
\end{abstract}

Keywords: multiple representations, level of detail, model generalisation, multiple representation database

\section{Introduction}

The change that may occur in geometry and topology of a feature when the level of detail (LoD) of its representation changes is termed as "multiple representations" (Buttenfield, 1993). The Multiple Representation Database (MRDB) is a database structure in which two or more representations of a same feature are stored individually at different LoDs and they are connected (Kilpeläinen, 1997).

The research problem on multiple representations was introduced by National Centre for Geographic Information and Analysis (NCGIA) (Buttenfield, 1993). Thereafter, many researchers have attempted this problem. Kilpeläinen (1997) proposed a two-fold model based on model \& cartographic generalisation and described its application in the context of multiple representation. The model for MRDB system was also presented and it was applied to topographic data. Chaudhry (2007) used a model generalisation approach to derive the geographic phenomena at 1:250,000 through aggregation process. This work involved the modelling of the geographic phenomena at multiple levels of detail.

The benefits of MRDB are non-redundant data, possibility to automatically check the consistency and error, flexible maintenance, etc. (Sarjakoski, 2007). These advantages of MRDB form the motivation for this research problem.
The remaining part of this paper is organised as follows. Section 2 describes the structure of MRDB. The methodology to derive MRDB is explained in section 3 . Lastly, conclusions and future work are provided in section 4 .

\section{Structure of MRDB}

The Figure 1 demonstrates the structure of the target MRDB. The plan is to use ladder approach to derive LoD2 $(1: 25,000)$ from LoD1 $(1: 10,000)$ and LoD3 (1:250,000) from LoD2 through model generalisation process. The strategy and the results of model generalisation process helps in maintaining the bidirectional links between LoDs as shown in Figure 1.

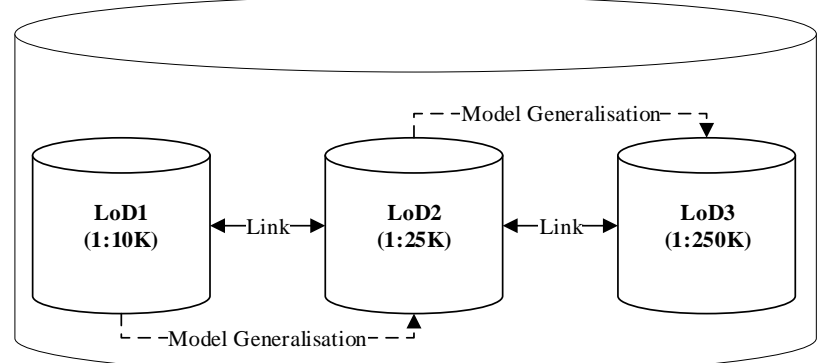

Figure 1. Multiple Representation Database (Boodala et al., 2018) 


\begin{tabular}{|l|l|l|l|l|l|}
\hline \multicolumn{2}{|c|}{ LoD1 (1:10K) } & \multicolumn{1}{c|}{ LoD2 (1:25K) } & \multicolumn{1}{c|}{ PoD3 (1:250K) } \\
\hline From & \multicolumn{1}{|c|}{ Product } & From & \multicolumn{1}{c|}{ Product } & From & Product \\
\hline OS & $\begin{array}{l}\text { OS Open Map }- \\
\text { Local (OML) }\end{array}$ & OS & $\begin{array}{l}\text { OS VectorMap District } \\
\text { (VMD) }\end{array}$ & OS & Strategi \\
\hline OS & $\begin{array}{l}\text { Boundary-Line } \\
\text { (BL) }\end{array}$ & OS & OS Open Roads (ORoads) & EG & EuroRegionalMap (ERM) \\
\hline & & ESDIN & ExM Large Scale (ExM-L) & ESDIN & $\begin{array}{l}\text { ExM Small or Medium Scale } \\
\text { (ExM-SM) }\end{array}$ \\
\hline
\end{tabular}

Table 1. Data products

\section{Methodology for MRDB Derivation}

\subsection{Data products used in modelling phase}

To design the MRDB structure at theme and feature level, the data products and their specifications listed in the Table 1 are used. These data products and specifications are obtained from organisations like Ordnance Survey (OS), EuroGeographics (EG) and also from European Spatial Data Infrastructure Network (ESDIN) project.

\subsection{Data models matching process}

The derivation of MRDB starts from the source database, i.e., LoD1. The OS Open Map - Local (OML) and Boundary-Line (BL) data products are used in the source database. The feature types and their geometries in the source database are shown in Table 2. (Ordnance Survey, 2016a, 2016b)

The feature types and their geometries which are supposed to be derived from LoD1 to populate target database, i.e., LoD2, are modelled using OS VectorMap District (VMD), OS Open Roads (ORoads) and ExM Large Scale (ExM-L). (ESDIN, 2010a; Ordnance Survey, 2016c, 2016d)

Similarly, for LoD3, Strategi, EuroRegionalMap (ERM) and ExM Small or Medium Scale (ExM-SM) are used. (BKG-Germany, 2017; ESDIN, 2010b; Ordnance Survey, 2015)

The Table 2 presents the one to one mapping of the feature types at different LoDs. This mapping is done using the definitions of feature types and their attribute information. This gives an idea of how to represent a feature at different LoDs and how to model their relationships. The feature type such as "Urban or BuiltupArea" in "Settlements" theme appears only at LoD3. This feature type can be modelled using "Building" feature type at LoD1 and LoD2. Thus, modelling the functional hierarchy.

\subsection{Generalisation operators and their order of applicability}

The model generalisation operators are essential to transform the database from LoD1 to other abstract levels (LoD2 and LoD3). The open data products (shown in Table 1) of different scales from OS are studied. This study helped in choosing the required model generalisation operators and also to decide their order of applicability. Table 3 is the outcome of this procedure and it will be refined after experiments by incorporating guidelines from ESDIN and/or EG.

The Add (C+), Eliminate (C-), Reclassify (Cc), Aggregate $(\mathrm{Gg})$, Collapse (Gc), Merge (Gm) and Simplify (Gs) (Roth et al., 2011) operators are used in this research. The definitions and notations of these operators given by Roth et al. (2011) are followed.

The Table 3 illustrates the generalisation process. To derive the 'Building' at LoD2, Cc followed by Gm operators are applied. This in turn is followed by Gc and Cc operators to derive LoD3. Similarly, it is shown for all the feature types in the source database (LoD1). The C+ operator is used to indicate the selection of feature types to LoD1.

The attribute information of the feature types is available in the form of codelists. Some codelists are hierarchical in nature and they can be used to drive the generalisation process. These codelists are used to form the classification hierarchies. This attribute information is used by $\mathrm{C}+, \mathrm{C}$ - and $\mathrm{Cc}$ operators. However, Gg, Gc, Gm and Gs operators use geometry information of the feature types.

The Figure 2 shows advantages of linking hierarchical codelists from different data models. In this example, the codelists from Infrastructure for Spatial Information in Europe (INSPIRE), ESDIN, EG, OS and European Location Framework (ELF) are used (BKG-Germany, 2017; ELF, 2016; ESDIN, 2010b; INSPIRE, 2013; Ordnance Survey, 2016b). The abstract level concept like 'Commerce and services' can be derived from the 'Secondary education' which is not possible by only using OS classification scheme. In the same way, other classification hierarchies can be linked.

\subsection{Geometric and topological constraints}

The geometric constraints which are essential to drive the generalisation are shown in Table 4. This information is organised according to LoDs and the sources of these values are also tabulated.

Kilpeläinen (1997) stated that "Topology does not change from scale to scale. An exception occurs only when the objects are omitted in context with scale transformation". In order to derive the consistent data at all LoDs, the topological relationships between the features at each LoD should be maintained. The topological constraints are defined at the feature level using ERM product specification (BKG-Germany, 2017) and it is shown in Table 5. 


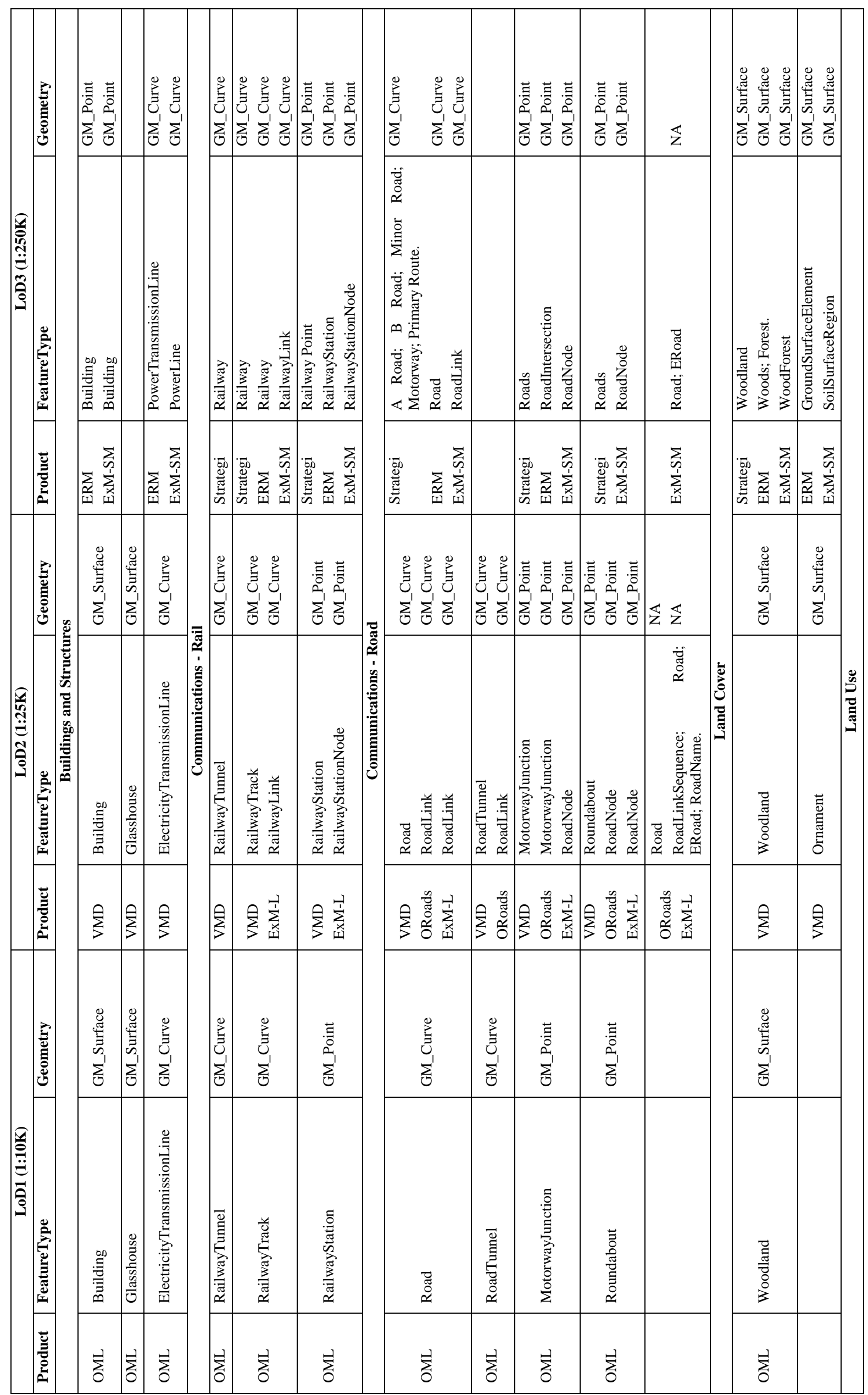




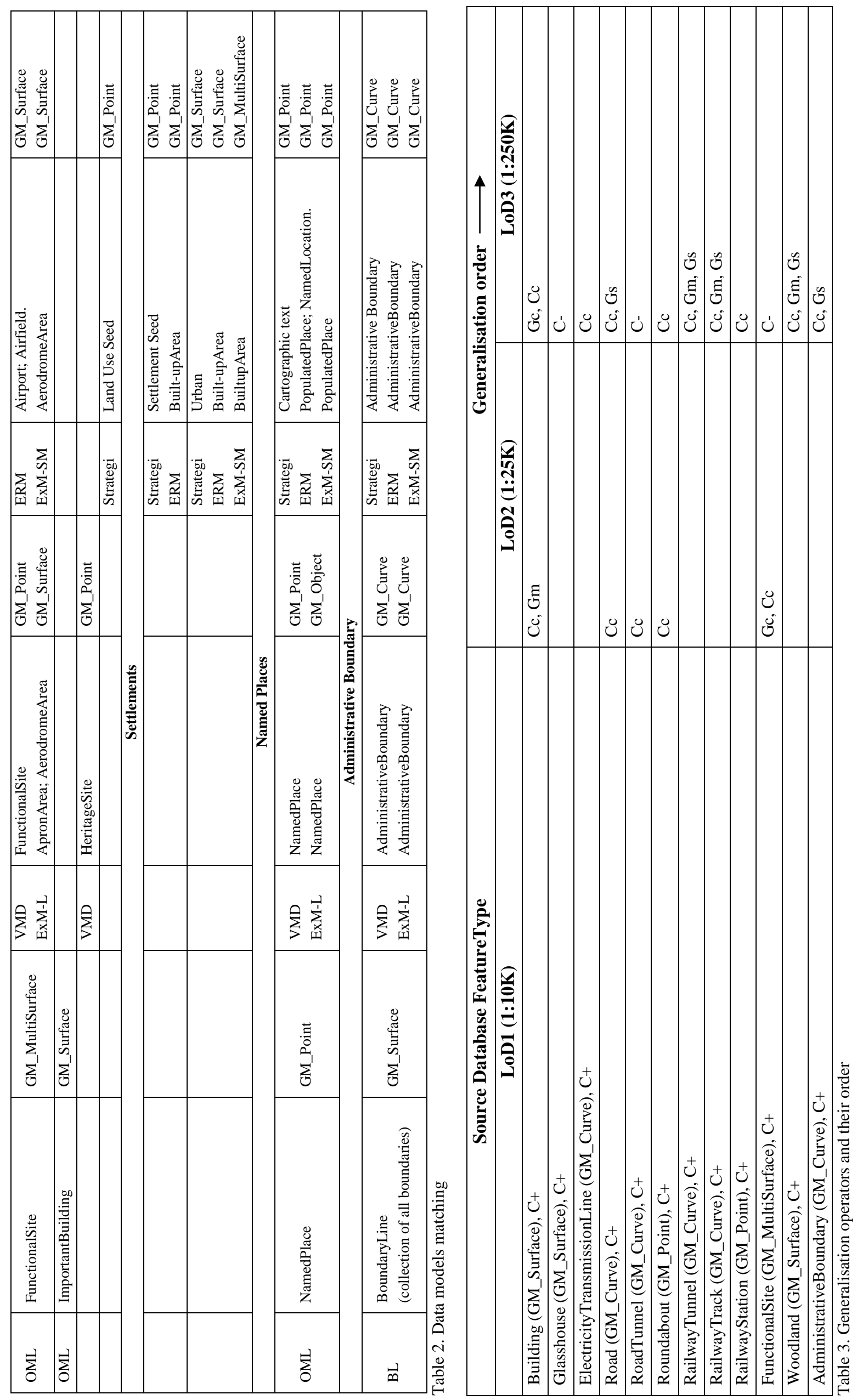

Proceedings of the International Cartographic Association, 2, 2019.

29th International Cartographic Conference (ICC 2019), 15-20 July 2019, Tokyo, Japan. This contribution underwent

single-blind peer review based on submitted abstracts. https://doi.org/10.5194/ica-proc-2-10-2019| @ Authors 2019. CC BY 4.0 License. 


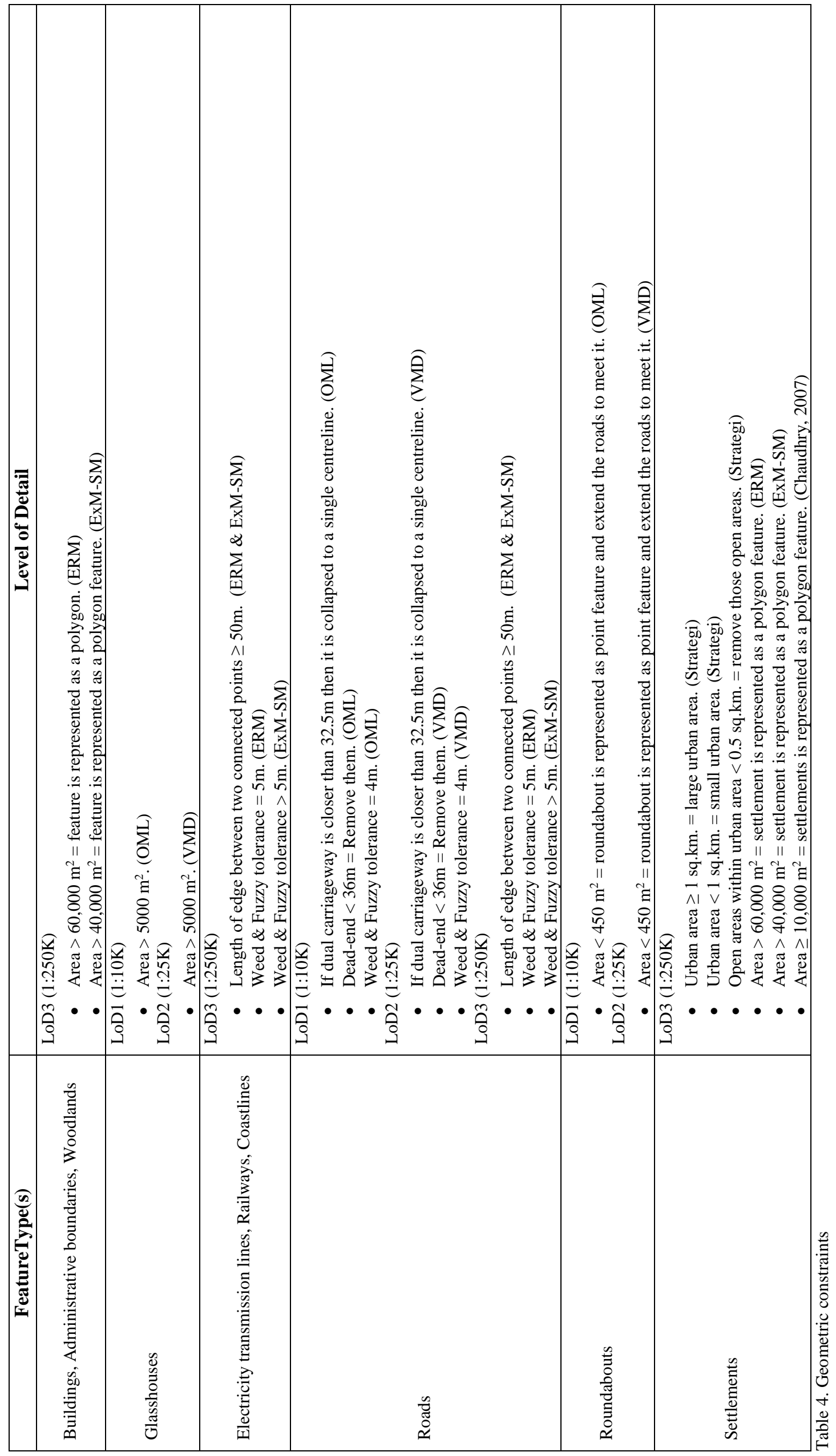

Proceedings of the International Cartographic Association, 2, 2019.

29th International Cartographic Conference (ICC 2019), 15-20 July 2019, Tokyo, Japan. This contribution underwent

single-blind peer review based on submitted abstracts. https://doi.org/10.5194/ica-proc-2-10-2019| @ Authors 2019. CC BY 4.0 License. 


\begin{tabular}{|c|c|c|c|c|c|c|c|c|c|c|c|c|c|c|c|c|c|c|c|c|c|}
\hline & 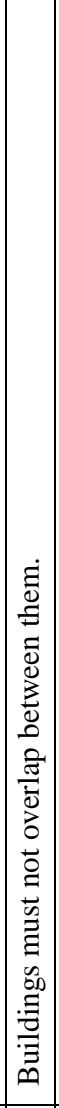 & 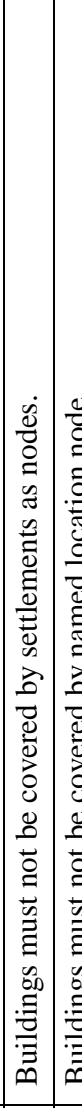 & 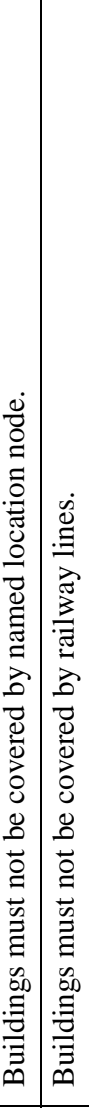 & 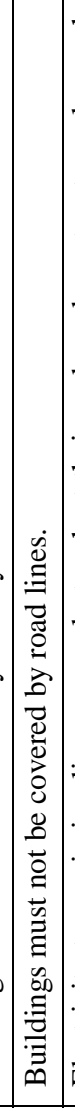 & 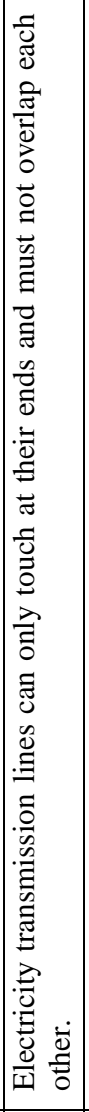 & 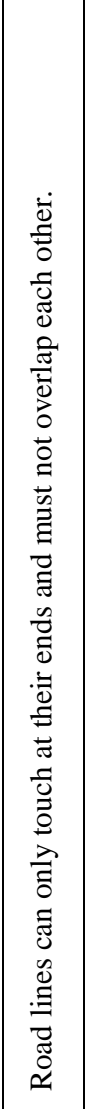 & 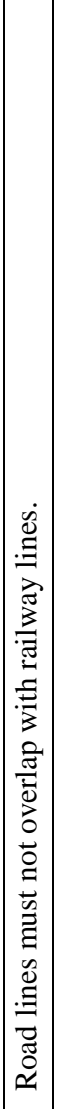 & 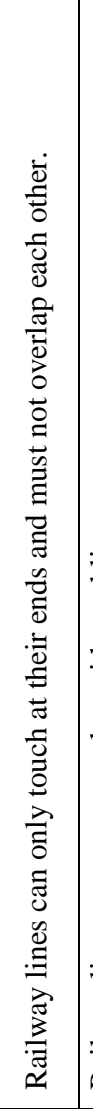 & 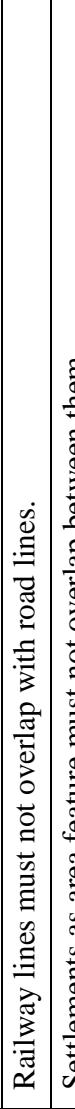 & 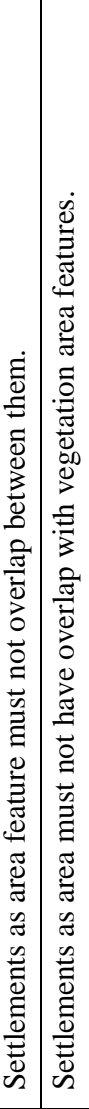 & 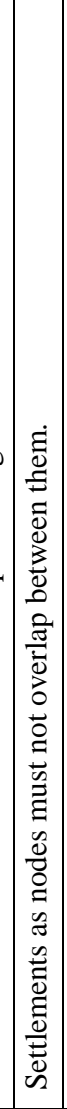 & 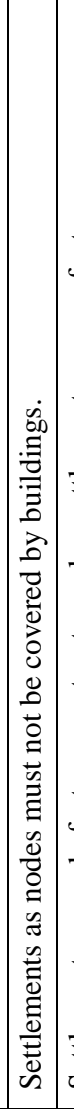 & 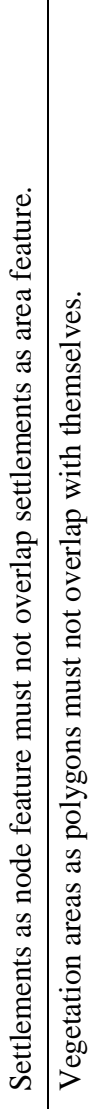 & 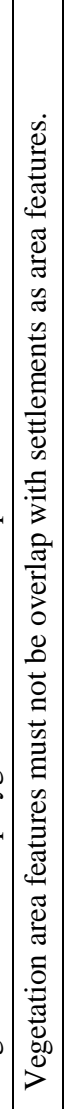 & 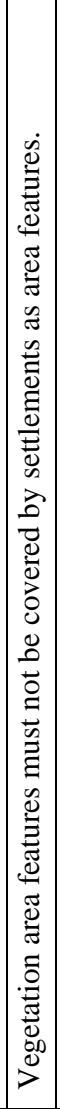 & 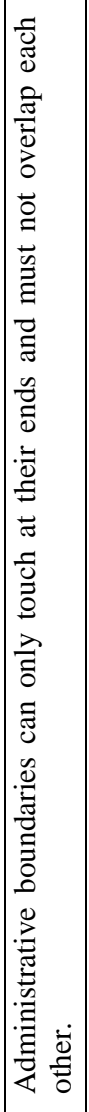 & 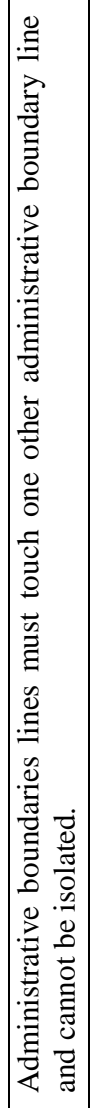 & 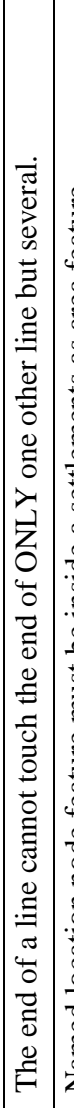 & 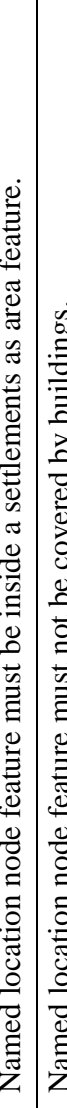 & 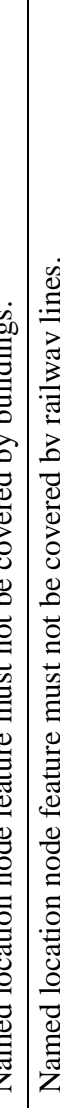 & \\
\hline & 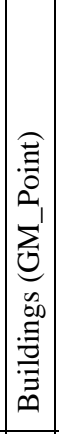 & 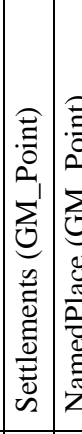 & 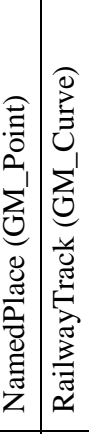 & 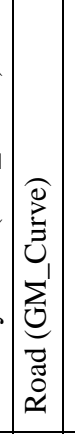 & & & 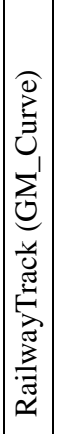 & & 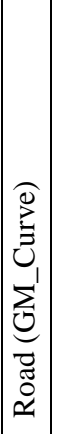 & 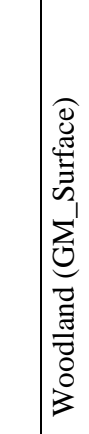 & & 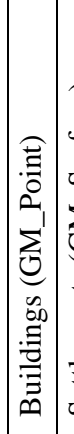 & 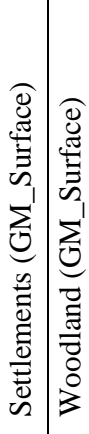 & 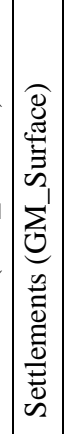 & 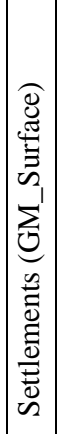 & & & & & 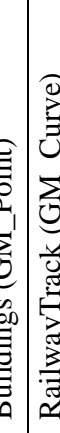 & \\
\hline 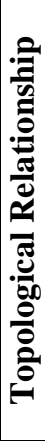 & 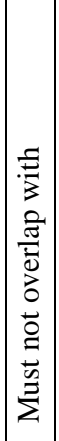 & 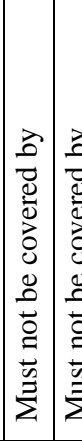 & 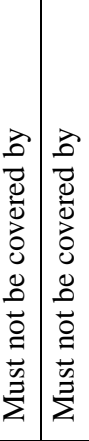 & 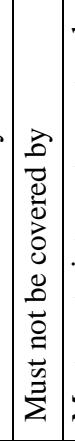 & 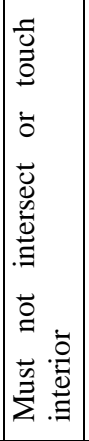 & 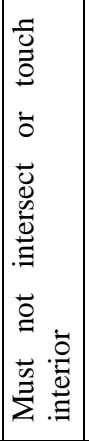 & 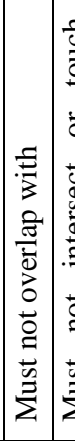 & 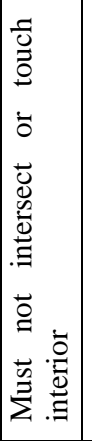 & 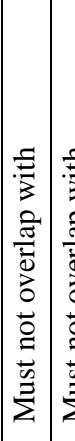 & 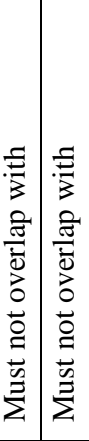 & 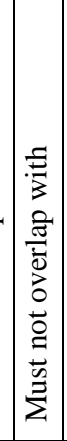 & 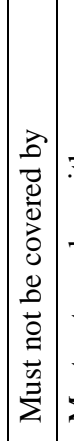 & 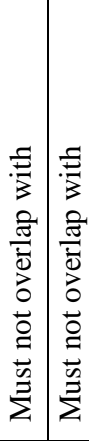 & 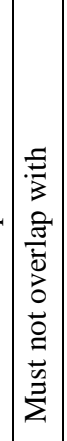 & 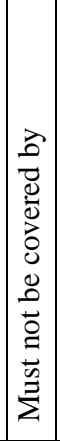 & 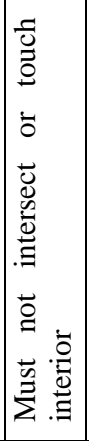 & 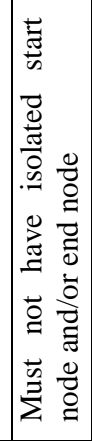 & 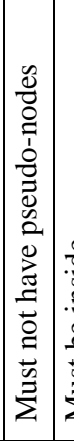 & & 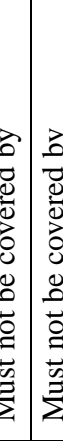 & \\
\hline 总 & & & 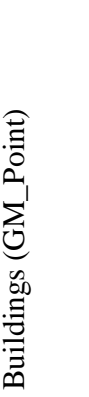 & & 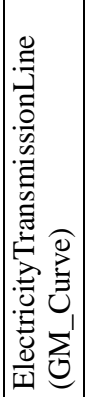 & 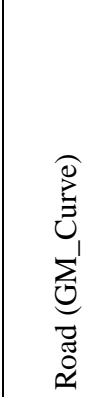 & & 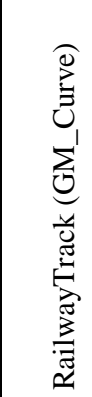 & & 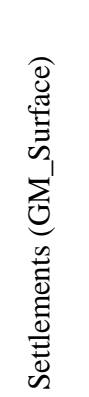 & & 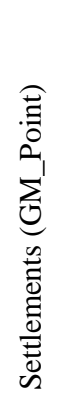 & & 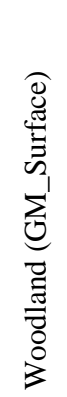 & & & 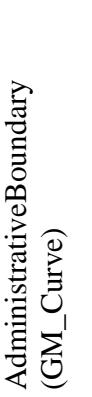 & & & & \\
\hline
\end{tabular}




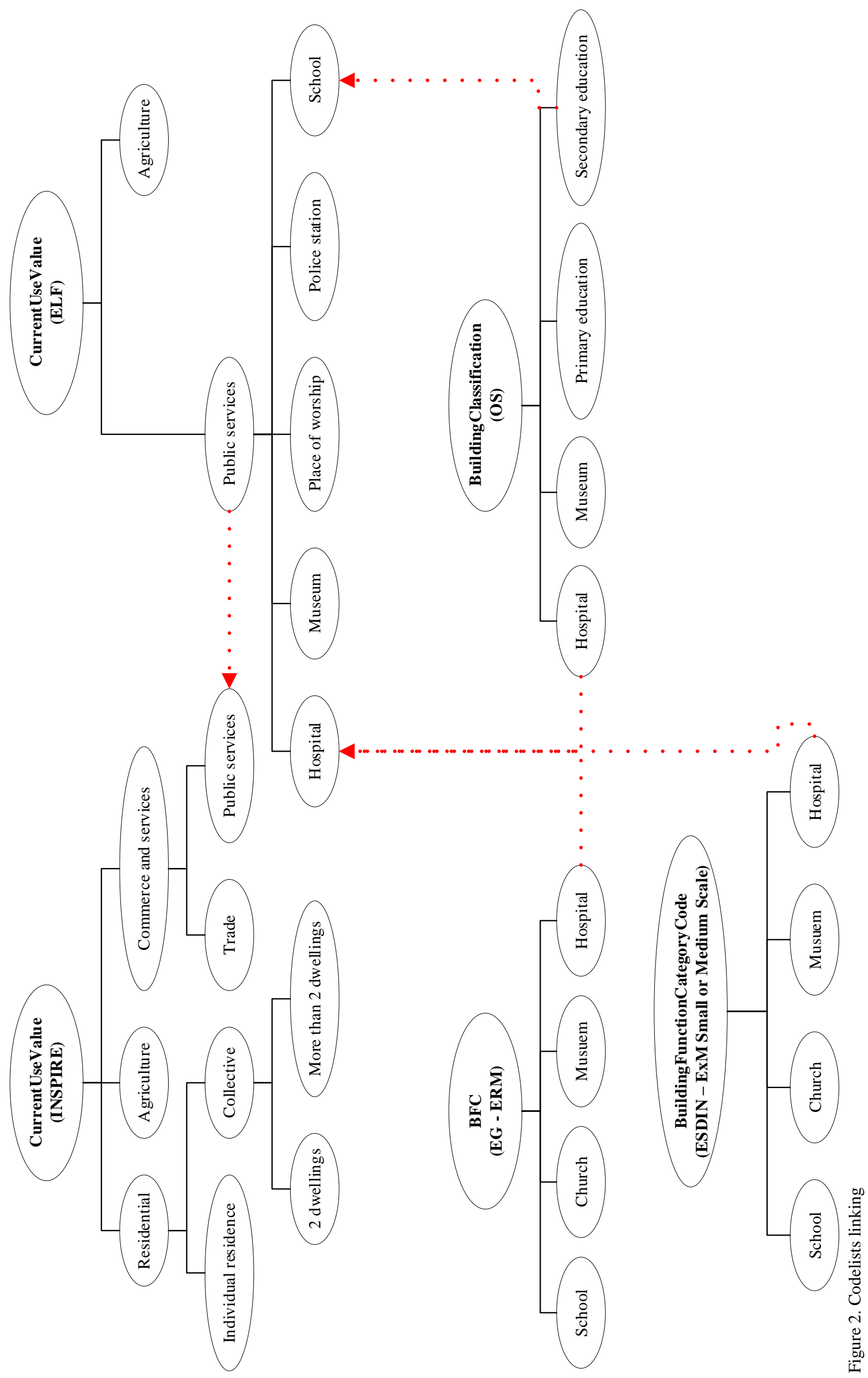

Proceedings of the International Cartographic Association, 2, 2019.

29th International Cartographic Conference (ICC 2019), 15-20 July 2019, Tokyo, Japan. This contribution underwent

single-blind peer review based on submitted abstracts. https://doi.org/10.5194/ica-proc-2-10-2019| @ Authors 2019. CC BY 4.0 License. 


\subsection{Selection of generalisation algorithms}

The model generalisation operators used in this research are implemented using the suitable generalisation algorithms. These algorithms for different feature types and contexts are chosen from the recommendations given in Automated Generalisation New Technology (AGENT) project reports (AGENT, 1999a, 1999b).

\subsection{Implementation and evaluation}

The research is currently in the implementation stage. The Python programming language is used to implement the proposed methodology and PostgreSQL with PostGIS extension is used as a backend database.

The OS OpenData products and their specifications are used to design the methodology. The results of the implementation will be tested against the same data products as well as the different products like OS Open Zoom Stack. The methodology will also be compared with the products of Survey of India (SoI).

\section{Conclusions}

This paper proposed the methodology to derive the MRDB. In line with that, the data modelling aspects for different LoDs, generalisation operators and their sequence, geometric and topological constraints are presented in this paper. This information is used to realise the prototype of the proposed concept. Eventually, the findings of this research will be transferred to derive MRDB for Indian scenario.

\section{Acknowledgements}

The authors are grateful to Ordnance Survey, United Kingdom for their OS OpenData products. The authors would also like to thank the anonymous reviewers for their suggestions.

\section{References}

AGENT (1999a). Selection of Basic Algorithms. (DD2): AGENT Consortium.

AGENT (1999b). Strategic Algorithms Using Organisations. (DD3): AGENT Consortium.

BKG-Germany (2017). EuroRegionalMap: PanEuropean Database at Medium Scale. (Specification and Data Catalogue for Data Production). BKG, Germany: EuroGeographics.

Boodala, J., Dikshit, O., \& Balasubramanian, N. (2018). Conceptual Framework for Multiple Representation Database. In : Vol. 38. Indian Cartographer, Emerging Technologies in Cartography. Indian National Cartographic Association.

Buttenfield, B. P. (1993). Multiple Representations: NCGIA Research Initiative 3. Buffalo NY.

Chaudhry, O. Z. (2007). Modelling Geographic Phenomena at Multiple Levels of Detail: A Model Generalisation Approach Based on Aggregation (PhD Thesis). The University of Edinburgh, Edinburgh.
ELF (2016). ELF Data Specification. (Data Specification for topographic and administrative reference data at all levels of detail): ELF Project.

ESDIN (2010a). ExM Data Specification - Large Scale. (Implementation Version, D7.2): EuroGeographics.

ESDIN (2010b). ExM Data Specification for Medium/Small scale. (Data Specification for topographic and administrative reference data at medium/small scale): EuroGeographics.

INSPIRE (2013). Data Specification on Buildings. (Technical Guidelines, D2.8.III.2): European Commission Joint Research Centre.

Kilpeläinen, T. (1997). Multiple Representation and Generalization of Geo-Databases for Topographic Maps (PhD Thesis). Suomen Geodeettisen Laitoksen julkaisuja. Geodeettinen Laitos., Kirkkonummi.

Ordnance Survey (2015). Strategi. (User Guide and Technical Specification). Southampton: Ordnance Survey, United Kingdom.

Ordnance Survey (2016a). Boundary-Line. (Product Guide and Technical Specification). Southampton: Ordnance Survey, United Kingdom.

Ordnance Survey (2016b). OS Open Map - Local. (Product Guide and Technical Specification). Southampton: Ordnance Survey, United Kingdom.

Ordnance Survey (2016c). OS Open Roads. (User Guide and Technical Specification). Southampton: Ordnance Survey, United Kingdom.

Ordnance Survey (2016d). OS VectorMap District. (User Guide and Technical Specification). Southampton: Ordnance Survey, United Kingdom.

Roth, R. E., Brewer, C. A., \& Stryker, M. S. (2011). A typology of operators for maintaining legible map designs at multiple scales. Cartographic Perspectives. (68), 29-64. https://doi.org/10.14714/CP68.7

Sarjakoski, L. T. (2007). Conceptual Models of Generalisation and Multiple Representation. In Generalisation of Geographic Information (pp. 1135). Elsevier. https://doi.org/10.1016/B978008045374-3/50004-1 